\title{
Monte Carlo calculation of the current-voltage characteristics of a two-dimensional lattice Coulomb gas
}

\author{
Hans Weber \\ Department of Physics, Luleå University of Technology, S-971 87 Luleå, Sweden \\ Mats Wallin \\ Department of Theoretical Physics, Royal Institute of Technology, S-100 44 Stockholm, Sweden \\ Henrik Jeldtoft Jensen \\ Department of Mathematics, Imperial College, London SW7 2BZ, United Kingdom \\ (Received 26 June 1995; revised manuscript received 27 November 1995)
}

\begin{abstract}
We have studied the nonlinear current-voltage characteristic of a two-dimensional lattice Coulomb gas by Monte Carlo simulation. We present three different determinations of the power-law exponent $a(T)$ of the nonlinear current-voltage characteristic, $V \sim I^{a(T)+1}$. The determinations rely on both equilibrium and nonequilibrium simulations. We find good agreement between the different determinations, and our results also agree closely with experimental results for $\mathrm{Hg}$-Xe thin-film superconductors and for certain single crystal thin-film high-temperature superconductors.
\end{abstract}

\section{INTRODUCTION}

In two dimensions the superconducting transition in zero magnetic field is a Kosterlitz-Thouless transition. ${ }^{1-3}$ This has been verified over the years in both experiments ${ }^{4}$ and in many models of superconductors like the $X Y$, Villain, and Coulomb gas models. ${ }^{1,5,6}$ The important degrees of freedom in a system undergoing a Kosterlitz-Thouless transition are thermally excited vortex pairs. The Kosterlitz-Thouless transition is sometimes also referred to as a vortex unbinding transition, as for temperatures below the transition temperature $T_{c}$ all vortices are bound in neutral pairs. These pairs start to unbind at and above $T_{c}$.

A typical way to look for a Kosterlitz-Thouless transition in experiments on thin superconducting films is to probe the current-voltage $(I V)$ characteristic., ${ }^{4,8}$ Both the linear and the nonlinear $I V$ characteristics have specific fingerprints identifying a Kosterlitz-Thouless transition. Vortices determine the $I V$ characteristic for the following reasons: If a vortex is dragged across the system a voltage is induced. Hence resistance is zero only if there are no vortices available to move across the system, and only then the system is truly superconducting. Vortices that are bound in neutral pairs are unable to move freely and to cause dissipation. However an external applied in-plane supercurrent yields a perpendicular Lorentz force acting in opposite direction on vortices with different vorticity. This gives a net flux of vortices across the system, which shows up as nonlinear (i.e., current-dependent) resistance.

Below the Kosterlitz-Thouless transition temperature all vortices are bound in neutral pairs by the logarithmic vortex interaction, and the linear resistance is thus zero. Therefore the system superconducts below the Kosterlitz-Thouless transition. The linear resistance drops to zero at the Kosterlitz-Thouless transition with an exponential functional form, $R \sim \xi^{-2}$ with $\ln \xi \sim\left|T-T_{c}\right|^{-1 / 2} .^{3}$ This is consistent with experiments, although the logarithm is a complication for quantitative comparison between theory and experiment. A finite applied current gives a power-law nonlinear $I V$ characteristic of the form $V \sim I^{a(T)+1}$. The critical current is thus zero. At the Kosterlitz-Thouless transition the $I V$ exponent $a(T)$ assumes the universal value 2 , so $V \sim I^{3}$ at $T=T_{c}$. For $T<T_{c}$ one has $a(T)>2$, and for $T>T_{c}$ one has $a(T)=0$ (for small enough currents). ${ }^{9}$ Experiments on, for example, thin $\mathrm{Hg}$-Xe alloy films ${ }^{4}$ and also for certain single-crystal hightemperature superconductors, ${ }^{7,8}$ among some, have confirmed this.

Since $I V$ characteristics are hard to calculate analytically computer simulation is a useful tool. IV characteristics of vortex systems have recently been calculated successfully with Monte Carlo simulations. ${ }^{10}$ Linear and nonlinear $I V$ characteristics of vortex glass superconductors have been reported in Refs. 10 and 11. In a recent Monte Carlo simulation of the Coulomb gas the linear resistance was used to locate the Kosterlitz-Thouless transition. ${ }^{12}$ The nonlinear $I V$ characteristics at the Kosterlitz-Thouless transition has been calculated in Ref. 13, and a finite-size scaling analysis accurately verified the relation $V \sim I^{3}$ at the Kosterlitz-Thouless transition.

In this paper we study the $I V$ characteristics of a lattice Coulomb gas model by Monte Carlo simulations of vortex dynamics. We calculate the $I V$ exponent $a(T)$ of the Coulomb gas in three different ways: (1) By direct Monte Carlo calculation of the nonlinear resistance, (2) by a selfconsistent linear screening construction for the energy barrier for current induced vortex-pair breaking giving thermally activated resistance, and (3) by a finite scaling construction from data for the linear resistance. All methods are based on Monte Carlo simulations, and we apply both equilibrium and nonequilibrium simulations. These three methods give the same results, giving us a consistent and simple picture of nonequilibrium response in this system. Furthermore, we 
compare our results for $a(T)$ with experiments. Scaling arguments give that $a(T)$ is a universal scaling function of a reduced Coulomb gas temperature $X=T / T_{c}$, and this is verified in experiments. ${ }^{3}$ We find close agreement between our Monte Carlo results and the experimental universal scaling curve. The agreement between different methods, and between our simulations and experiments, are the main results of our paper. Some of our Monte Carlo results for the nonlinear $I V$ characteristics have been obtained previously, ${ }^{13}$ as explained above.

The paper is organized as follows: In Sec. II we define the lattice Coulomb gas model. In Sec. III we study various approaches to the $I V$ characteristics. In Sec. IV we describe our Monte Carlo methods for calculating $I V$ characteristics. In Sec. V we present the Monte Carlo results. Section VI contains discussion and conclusions.

\section{LATTICE COULOMB GAS}

A useful starting point for calculations with superconductors in the presense of currents and fields is the GinsburgLandau model, with the order parameter $\Psi(\mathbf{r})=|\Psi(\mathbf{r})| e^{i \phi(\mathbf{r})}$ describing the superconducting order of the system. However, this model does not focus particularly on vortex degrees of freedom. The vortices constitute the essential degrees of freedom near the Kosterlitz-Thouless transition. An approximation to the Ginsburg-Landau model which focuses only on the vortices is given by the Coulomb gas model. Here thermal fluctuations in the magnitude of $\Psi$ are neglected, since they are relevant only close to the mean-field transition temperature, which is assumed to be well above the vortex transition temperature $T_{c}$. In our simulations the model is discretized and put on a lattice. The approximation made in the lattice discretization will only affect the short-range behavior of the vortices, as the lattice defines the smallest possible separation. The critical properties will however not be effected. In general, large length scale properties should be reasonable modeled by the lattice Coulomb gas close to $T_{c}$.

The lattice Coulomb gas ${ }^{14,15}$ is defined by the partition function $Z$ on a square lattice of side length $L$ using periodic boundary conditions:

$$
\begin{gathered}
Z=\operatorname{Tr}_{n} \exp [-\beta(H-\mu N)], \\
H=\frac{1}{2} \sum_{i, j} n_{i} G_{i j} n_{j}, \\
N=\sum_{i}\left|n_{i}\right|,
\end{gathered}
$$

where $H$ is the Hamiltonian, $n_{i}$ is the vorticity at site $i$ (Coulomb gas charge), $\mu=-E_{c}$ is the vortex "chemical potential" and $E_{c}$ is the vortex core energy, and $T=1 / \beta$ is the Coulomb gas temperature. ${ }^{3}$ The trace is over $n_{i}=0, \pm 1$ on all sites $i$, subject to overall neutrality, $\Sigma_{i} n_{i}=0 . G_{i j}$ is the lattice Green's function for the logarithmic two-dimensional (2D) vortex interaction,

$$
G_{i j}=\frac{1}{L^{2}} \sum_{\mathbf{k}} \frac{\pi e^{i \mathbf{k} \cdot\left(\mathbf{r}_{i}-\mathbf{r}_{j}\right)}}{2-\cos \left(k_{x}\right)-\cos \left(k_{y}\right)},
$$

where $\mathbf{k}$ are the reciprocal-lattice vectors, $k_{x}, k_{y}=2 \pi n / L, n=0, \ldots, L-1$.

We will calculate the response voltage to an applied current imposed on the Coulomb gas. The above definition does not include any net currents. How to include them and to calculate $I V$ characteristics by Monte Carlo simulation is described in the next section.

\section{CURRENT-VOLTAGE CHARACTERISTICS}

In this section we discuss various aspects and approaches to the current-voltage characteristics of 2D superconductors close to the Kosterlitz-Thouless transition.

\section{A. Linear resistance}

A basic experiment on a superconductor is to measure the linear resistance. Such measurements on thin films of both conventional low- $T_{c}$ superconductors ${ }^{4}$ and single-crystal high- $T_{c}$ materials, ${ }^{7,8}$ have been successfully interpreted in terms of thermally excited vortex fluctuations analyzed by use of the Coulomb gas. ${ }^{3}$

The linear resistivity is defined by $\rho=E / j$ for $j \rightarrow 0$, where $j$ is the applied supercurrent density and $E$ is the resulting induced electric field. Some words about notation: Since resistance and resistivity have the same dimension in two dimensions and our system is homogeneous, they are the same, and they we will both be denoted by $R . R$ will be reserved for linear resistance, and will not be used to denote nonlinear resistance. An applied supercurrent is denoted by $I=j L$, and voltage is $V=E L$.

To determine the linear resistance in simulations of the Coulomb gas from $E / j$ for small $j$ has its limitations, as we have to repeat the calculation at a sequence of current densities $j$, to make sure that $j$ is small enough to be in the linear regime. If the purpose is to measure only the linear resistance, and not $E$ as function of $j$, a different approach is to use the Nyquist formula, ${ }^{16}$ which relates the linear resistance to the equilibrium voltage fluctuations:

$$
R=\frac{1}{2 T} \int_{-\infty}^{+\infty} d t\langle V(t) V(0)\rangle,
$$

where $V(t)$ is the induced voltage from vortex motion at time $t$. As an alternative to Eq. (5) the Kubo formula for the vortex currents $I_{v}, R=1 / 2 T \int_{-\infty}^{\infty} d t\left\langle I_{v}(t) I_{v}(0)\right\rangle$ can be used. Given the Josephson relation we see immediately that the Kubo formula equals the Nyquist relation.

The linear resistance has been successfully used in a simulation $^{12}$ to locate the Kosterlitz-Thouless transition temperature $T_{c}$ of the 2D lattice Coulomb gas. They find the finite-size scaling relation at $T_{c}$ :

$$
L^{2} R\left(1+\frac{1}{4 \ln (L)+C}\right)=\text { const at } T=T_{c},
$$

to be valid to a very high precision.

The scaling relation Eq. (6) was derived from the following argument. We assume the dynamical exponent $z=2$ for free vortex diffusion in two dimensions. ${ }^{13,17}$ The linear resistance is a dynamical quantity, it relates to the correlation time $\tau$, which at $T_{c}$ diverges like $\tau \sim \xi^{z}$, where $\xi$ is the 
correlation length. According to the Josephson relation the voltage $V \sim d \Delta \phi / d t \sim \tau^{-1}$, where $\Delta \phi$ is the gradient of the phase of the Ginsburg-Landau order parameter. ${ }^{18}$ Therefore, we expect the linear resistance, Eq. (5), to scale like $R \sim \xi^{-2}$ at $T_{c}$. At $T_{c}$ the correlation length diverges and is cut of by the finite size $L$ of the lattice and hence $R L^{2}=$ const at $T_{c}$, to lowest order. The scaling relation has a logarithmic correction which has been included in Eq. (6). This correction is readily obtained from the corresponding correction terms for $1 / \epsilon$ and $\lambda .{ }^{19}$

\section{B. Thermally activated resistance}

The above scaling argument led to a finite-size scaling formula which is useful for locating the transition temperature from Monte Carlo data for the resistance of finite samples. Here we will do a more detailed analysis that will also lead to the same formula. The analysis here does not directly involve scaling arguments, but considers the interactions between vortices in the Coulomb gas. The analysis will give expressions for the resistance from thermally activated free vortices in the Coulomb gas in the presence of an applied supercurrent. This more detailed analysis will be useful in later sections when we analyze Monte Carlo data for the Coulomb gas.

According to the Josephson relation the voltage $V$ caused by vortex motion is

$$
V \sim \frac{d \Delta \phi}{d t} \sim n_{F} I,
$$

where we assume that the resistance is proportional to the density of "free" vortices, $n_{F}$, defined by the the DebyeHückel relation. The linear resistance $R$ is defined by the limit of zero current $I$ :

$$
R=\lim _{I \rightarrow 0} \frac{V}{I} \sim n_{F} .
$$

To make an estimate of the density of free vortices we proceed by the following simple model. The energy $E(r)$ of a vortex pair of separation $r>r_{0}$ in the presence of a current $I$ is $^{17}$

$$
E(r)=E_{0}+E_{1} \ln \left(\frac{r}{r_{0}}\right)-I\left(r-r_{0}\right),
$$

where $E_{0}$ is a constant, $E_{1}$ is discussed below and $r_{0}$ is the smallest possible separation, which we will set to $r_{0}=1$ from now.

We will now use the linear screening approximation ${ }^{3}$ to derive an expression for the second term $E_{1} \ln r$ in Eq. (8). The expression is obtained from the Fourier transform of the linearly screened potential $V_{l}(k)$,

$$
V_{l}(k)=-\frac{1}{\tilde{\boldsymbol{\epsilon}}(k)} \frac{2 \pi}{k^{2}+\lambda^{-2}} .
$$

Here $\lambda$ is the vortex screening length, and $\tilde{\boldsymbol{\epsilon}}(k)$ is the part of the dielectric function, $\epsilon(k)$, describing the polarization of the bound pairs. The two $\epsilon$ are related by

$$
\frac{1}{\epsilon(\mathbf{k})}=\frac{1}{\tilde{\epsilon}(\mathbf{k})} \frac{k^{2}}{k^{2}+\lambda^{-2}}
$$

In the limit $\lambda \rightarrow \infty$ the two different dielectric functions become equal. This is the case for temperatures below $T_{c}$. The dielectric function, $1 / \epsilon(k)$, is obtained from the charge fluctuations below in Eq. (24). The real-space expression for $E(r)$ is obtained from

$$
E(r)=\lim _{\lambda \rightarrow \infty} V_{l}(r),
$$

where

$$
V_{l}(\mathbf{r})=\int \frac{d \mathbf{k}_{2}}{2 \pi} V_{l}(\mathbf{k}) e^{i \mathbf{k} \cdot \mathbf{r}}
$$

We can obtain an approximate expression for $V_{l}(r)$ by making use of the fact that $\tilde{\boldsymbol{\epsilon}}(\mathbf{k})$ only depends weakly (in most of $k$ space) on $\mathbf{k}$. For a given distance $r$, the Fourier integral picks up its main contribution from the $k$ values around $2 \pi / r$. Hence

$$
V_{l}(r)-V_{l}(r=1) \approx-\frac{1}{\tilde{\epsilon}(k=2 \pi / r)} K_{0}(r / \lambda) .
$$

Here we have subtracted $V_{l}(r=1)$ in order to eliminate the creation energy. $K_{0}$ denotes a modified Bessel function. As $\lambda \rightarrow \infty$ this expression reduces to

$$
V_{l}(r)-V_{l}(r=1) \approx \frac{1}{\epsilon(2 \pi / r)} \ln (r / \lambda),
$$

where we use $\epsilon$ instead of $\tilde{\epsilon}$, as the temperature is below $T_{c}$. by $^{20}$

According to this discussion the coefficient $E_{1}$ is given

$$
E_{1}=\frac{1}{\epsilon(2 \pi / r)} .
$$

The weak $r$ dependence describes the effect of the surrounding vortex pairs. The coefficient $E_{0}$ contains the remaining constant terms from Eq. (9). In a first approximation we will neglect the $r$ dependence in $E_{1}$.

The energy $E(r)$ in Eq. (8) has a maximum at separation $r^{*}=E_{1} / I$ and the energy needed to separate a vortex pair to this distance is ${ }^{4}$

$$
\begin{gathered}
\Delta E=E\left(r^{*}\right)-E(r=1)=E_{1} \ln \left(r^{*}\right)-I\left(r^{*}-1\right), \\
\Delta E=E_{1} \ln \left(\frac{E_{1}}{I}\right)-E_{1}+I .
\end{gathered}
$$

Let $\Gamma$ denote the thermal production rate of free vortices. A vortex vanishes when it collides with an antivortex. Hence, it appears reasonable to assume an annihilation rate proportional to $n_{F}^{2}$. This leads to the following rate equation:

$$
\dot{n}_{F}=\Gamma-c n_{F}^{2} .
$$


Where $c$ is a constant. The steady-state condition is $\dot{n}_{F}=0$ and hence we have $\Gamma \propto \sqrt{n_{F}}$. Assuming that $\Gamma$ is determined by activation over the barrier $\Delta E$ we get the following production rate: ${ }^{17}$

$$
\Gamma \propto e-\Delta E / T
$$

and hence for the resistance $R$ from Eq. (7),

$$
\begin{aligned}
R \propto n_{F} \propto e^{-\Delta E / 2 T} & \propto \exp \left\{-\frac{1}{2 T}\left[E_{1} \ln \left(\frac{E_{1}}{I}\right)-E_{1}+I\right]\right\} \\
& \propto\left(\frac{E_{1}}{I}\right)^{-E_{1} / 2 T} e^{E_{1} / 2 T} e^{-I / 2 T} .
\end{aligned}
$$

Keeping the important term for small but finite $I$ we arrive at

$$
R \propto\left\langle\frac{E_{1}}{I}\right\rangle^{-E_{1} / 2 T} .
$$

A given current $I$ gives rise to a "current length scale" $r^{*}$ from the maximum condition in Eq. (8). As the lattice of the system has a finite size, this sets an upper limit to the "current length" and hence a lower limit to the current producing nonlinear resistance. The smallest current giving nonlinear resistance is $I^{*}=E_{1} / r^{*}$ with $r^{*}=L$ and hence for currents smaller than $I^{*}$ the resistance will be cut off by the finite size $L$ of the lattice and the resistance becomes Ohmic. The Nyquist resistance is calculated with $I=0$ and hence

$$
R \propto\left\langle\frac{1}{L}\right\rangle^{-E_{1} / 2 T} .
$$

This means that we can scale the linear resistance $R$ from the Nyquist relation Eq. (5) with the exponent $E_{1} / 2 T$. This exponent is precisely $a(T)$, the exponent of the nonlinear $I V$ characteristics [see Eq. (18) below], hence

$$
f(T)=R L^{a(T)}
$$

should collapse onto a single curve for different lattice sizes $L$. I.e., $f(T)$ should not depend on lattice size $L$. The resistance we use for this scaling will be the one determined from the voltage fluctuations Eq. (5). The exponent determined from resistance data at zero current will be denoted $a_{R}(T)$.

\section{Nonlinear $I V$ exponent}

We are going to make use of a couple of different expressions for the power-law exponent $a(T)$ of the nonlinear $I V$ characteristics. From Eq. (15) we get the nonlinear $I V$ characteristic,

$$
V \propto\left\langle\frac{E_{1}}{I}\right\rangle^{-E_{1} / 2 T} I \propto I^{a(T)+1} .
$$

The exponent calculated by monitoring the voltage response $V$ as a function of an applied supercurrent $I$ will be denoted $a_{I V}(T)$. On a finite system we will obtain a nonlinear voltage response only above a finite applied current, given by $I^{*} \sim E_{1} / L$, such that the current length $r^{*}$ is shorter than the size $L$ of the system, as discussed above.

\section{Self-consistent $I V$ characteristic}

Another expression for the $I V$ characteristic is obtained if we include the $r$ dependence in $E_{1}$ in Eq. (10). The length dependence can in a first approximation [in an expansion in derivatives of $\left.E_{1}(r)\right]$ be included simply by replacing $E_{1}$ in Eq. (15) by $1 / \epsilon\left(2 \pi / r^{*}\right)$ in the extremum equation $I=E_{1} / r^{*}$. Our rationale for this choice is that at the separation $r^{*}$ the vortex pair is broken apart and we therefore use the stiffness $1 / \epsilon(r)$ of the system at this separation. We find the appropriate $\epsilon(r)$ by solving self-consistently the equation

$$
I=\frac{1}{\epsilon\left(2 \pi / r^{*}\right) r^{*}}=\frac{k^{*}}{\epsilon\left(k^{*}\right) 2 \pi} .
$$

The self-consistent $\epsilon$ obtained by solving Eq. (19) will be denoted $\epsilon^{*}$. The relation between the exponent $a(T)$ and the dielectric function $\tilde{\epsilon}$ is according to Eqs. (15) and (10) given by the expression (see Ambegoakar et al. ${ }^{17}$ )

$$
a(T)_{\mathrm{AHNS}}=\frac{1}{2 T \epsilon^{*}} ;
$$

here we use $\epsilon^{*}$ as we are at temperatures below $T_{c}$.

Recently Minnhagen et al. have used scaling arguments to derive an alternative relationship between $a(T)$ and $\epsilon$, given by $^{21}$

$$
a(T)_{\mathrm{PM}}=\frac{1}{T \epsilon^{*}}-2 .
$$

As one immediately realizes Eq. (21) is not consistent with the activation argument used to derive Eq. (20). In order to reconcile Eq. (21) with a rate equation like Eq. (13) Minnhagen et al.. have made the following suggestion. They assume that the activation is correctly represented by $\Gamma$ in Eq. (14). The recombination, which in Eq. (13) is represented by the innocently looking term $n_{F}^{2}$, is on the other hand supposed to be replaced by $n_{F}^{1+b}$ with $b=2 /\left(E_{1} / T-2\right)$. The sole argument for this replacement is unfortunately so far simply the observation that one then can derive Eq. (21) from an equation like Eq. (13). Nonetheless, we shall see below that for temperatures below $T_{c}$ Eq. (21) fits the simulation data much better than Eq. (20) does. However a motivation for a recombination term different from the one in Eq. (13) has not been presented. At $T_{c}$ both relations reproduce the same exponent $a\left(T=T_{c}\right)=2$.

\section{MONTE CARLO SIMULATION}

In this section we describe how we calculate currentvoltage characteristics by Monte Carlo simulation of the lattice Coulomb gas. The algorithm to simulate the lattice Coulomb gas works as follows: ${ }^{15}$ First we pick a nearestneighbor pair $(i, j)$ of lattice sites at random. Then we try to increase $n_{i}$ by one and to decrease $n_{j}$ by one, thus preserving overall vortex neutrality, $\Sigma_{i} n_{i}=0$. This Monte Carlo move of inserting a neutral pair will be interpreted as transfer of one unit vortex from site $j$ to $i$. If the energy change is $\Delta E$ we accept this trial move according to the standard Metropolis algorithm $^{22}$ with probability $\exp (-\Delta E / T)$. These simple Monte Carlo moves can both create, annihilate, and move vortices. Thermodynamic averages are computed as Monte 
Carlo time averages over the sequence of generated configurations.

To calculate $I V$ characteristics works as follows: ${ }^{10,11}$ An applied current density $j$ gives a Lorentz force of $j h /(2 e)$ on a unit vortex. The Lorentz force can be incorporated in the Monte Carlo moves ${ }^{10}$ by adding to $\Delta E$ an extra term $j h /(2 e)$ if the unit vortex moves in the direction opposite to the Lorentz force, subtracting this term if it moves in the same direction, and making no change in $\Delta E$ if it moves in a perpendicular direction. Biasing the Monte Carlo moves in this way takes the system out of equilibrium and causes a net flux of vortices in a direction perpendicular to the current. This generates a voltage given by the Josephson relation:

$$
V=\frac{h}{2 e}\left\langle I_{v}(t)\right\rangle
$$

where $I_{v}(t)$ is the vortex current. Here $t$ denotes Monte Carlo time, incremented by $\delta t$ after each attempted move. The vortex current is $I_{v}(t)=+1 / L \Delta t$ if a unit vortex moves one lattice spacing in the direction of the Lorentz force at time $t$, and $I_{v}(t)=0$ otherwise. We use units such that $\Delta t=1 / L^{2}$ so that an attempt is made to move a vortex on each lattice site, on average, per unit time. We also use units such that $h /(2 e)=1$.

The linear resistance can also be obtained from the Nyquist relation in Eq. (5) for equilibrium voltage fluctuations in the absence of any net currents. For discrete Monte Carlo time it is given by, ${ }^{16,23}$ in our units,

$$
R=\frac{1}{2 T} \sum_{t=-\tau}^{\tau} \Delta t\langle V(t) V(0)\rangle
$$

The cutoff time $\tau$ is set to 1000 time steps, this has proved to be sufficient as $R$ saturates for values of $\tau$ less than 100 time steps.

The underlying assumption in using Monte Carlo dynamics to calculate $I V$ characteristics is that Monte Carlo time can be equated with real time. This approximation has proven reasonable in other simulations of vortex dynamics. ${ }^{10,11,13}$ It should be good near a critical point where vortex motion is slow and overdamped, but not so satisfactory at high temperatures or currents where the discreteness of Monte Carlo time becomes visible as a saturation of vortex velocities. We get strong support for this assumption from the results in the next section since we can reproduce the expected $I V$ characteristic at the Kosterlitz-Thouless transition, and since we come close to experiments. One can, in principle, also test this by comparison to dynamics simulations where time evolution equations are integrated. ${ }^{24}$

In the equilibrium simulations in the case of no net currents we typically use $10^{6}-10^{7}$ Monte Carlo sweeps (one sweep means one Monte Carlo time step defined above, i.e., $L \times L$ attempts to insert nearest-neighbor pairs), and in the nonequilibrium case of an applied current we typically use $10^{5}-10^{6}$ Monte Carlo sweeps. In the evaluation of the Nyquist formula for the linear resistance, Eq. (5), we typically sum over $10^{3}-10^{4}$ time steps.

The first task for our simulations is to locate the Kosterlitz-Thouless transition temperature $T_{c}$. The usual universal jump criterion for a Kosterlitz-Thouless transition involves the dielectric response function $1 / \epsilon$, given by

$$
\begin{gathered}
\frac{1}{\epsilon(\mathbf{k})}=1-\frac{2 \pi}{k^{2} T L^{2}}\left\langle n_{\mathbf{k}} n_{-\mathbf{k}}\right\rangle, \\
n_{\mathbf{k}}=\sum_{\mathbf{r}_{i}} n_{i} e^{-i \mathbf{k} \cdot \mathbf{r}_{i}},
\end{gathered}
$$

where $n_{\mathbf{k}}$ is the Fourier transform of the vortex density. The limit $\mathbf{k} \rightarrow 0$ denoted $1 / \epsilon(\mathbf{k}=0)$, corresponds to the fully renormalized long-wavelength superfluid density, and the universal jump criterion tells us that $1 / \epsilon(\mathbf{k}=0)$ jumps from $4 T_{c}$ at $T=T_{c}^{-}$to 0 at $T=T_{c}^{+} \cdot{ }^{9}$ A practical difficulty for locating $T_{c}$ from Monte Carlo data on small lattices with this procedure is that extrapolation to the $\mathbf{k}=0$ limit requires large lattices, as the smallest nonzero $\mathbf{k}$ is $2 \pi / L$. The corresponding quantity to $1 / \epsilon(\mathbf{k}=0)$ in the two-dimensional $X Y$ model is called the helicity modulus $\gamma \cdot{ }^{5}$ Both quantities have been used to locate the Kosterlitz-Thouless transition temperature in Monte Carlo calculations. ${ }^{5,25,19}$

In the data analysis in the next section we use an alternative procedure to locate $T_{c}$ from the linear resistance. ${ }^{12} \mathrm{We}$ obtain the linear resistance $R$ from the Nyquist formula in Eq. (5) for a sequence of system sizes $L$ and temperatures $T$. According to Eq. (6) data for $L^{2} R$ for different system sizes should become system size independent at the critical temperature, which is our criterion to locate $T_{c}$. This circumvents the difficulties of using $1 / \epsilon(\mathbf{k}=0)$. The two determinations give within error bars the same value for $T_{c}$.

\section{RESULTS}

We present Monte Carlo simulation results for three different determinations of the $I V$ exponent $a(T)$ for the 2D Coulomb gas model. The results we show here are for the chemical potential $\mu=0.0$ and lattice size $L=32$ if not differently stated.

\section{A. Nonlinear $I V$ exponent}

Our first method consists in a direct measurement of the electric field $E$ induced by an applied current density $j$. In Fig. 1(a) results for the $I V$ characteristic of the twodimensional Coulomb gas are shown. The dashed line in the $\ln (E)$ versus $\ln (j)$ plot has slope three and represents the slope at $T=T_{c}$ according to the universal jump condition. ${ }^{9}$ The solid curves represent results for different temperatures. For very high current the voltage response saturates. This is because when all attempts to move the vortices in the direction of the Lorentz force are already accepted, further increasing the current cannot give more voltage. For low enough current there is a crossover to Ohmic resistance, when the current length equals the system size, and the nonlinear dependence of the resistance on the current vanishes. The regime where we probe the nonlinear $I V$ characteristic is for this figure approximately from $\ln j \approx-1.5$ up to $\approx-0.5$. According to the Kosterlitz-Thouless theory the slope of the lines should be 3 at the critical temperature, and this criteria can be used to determine $T_{c}$. We will however use an inde- 

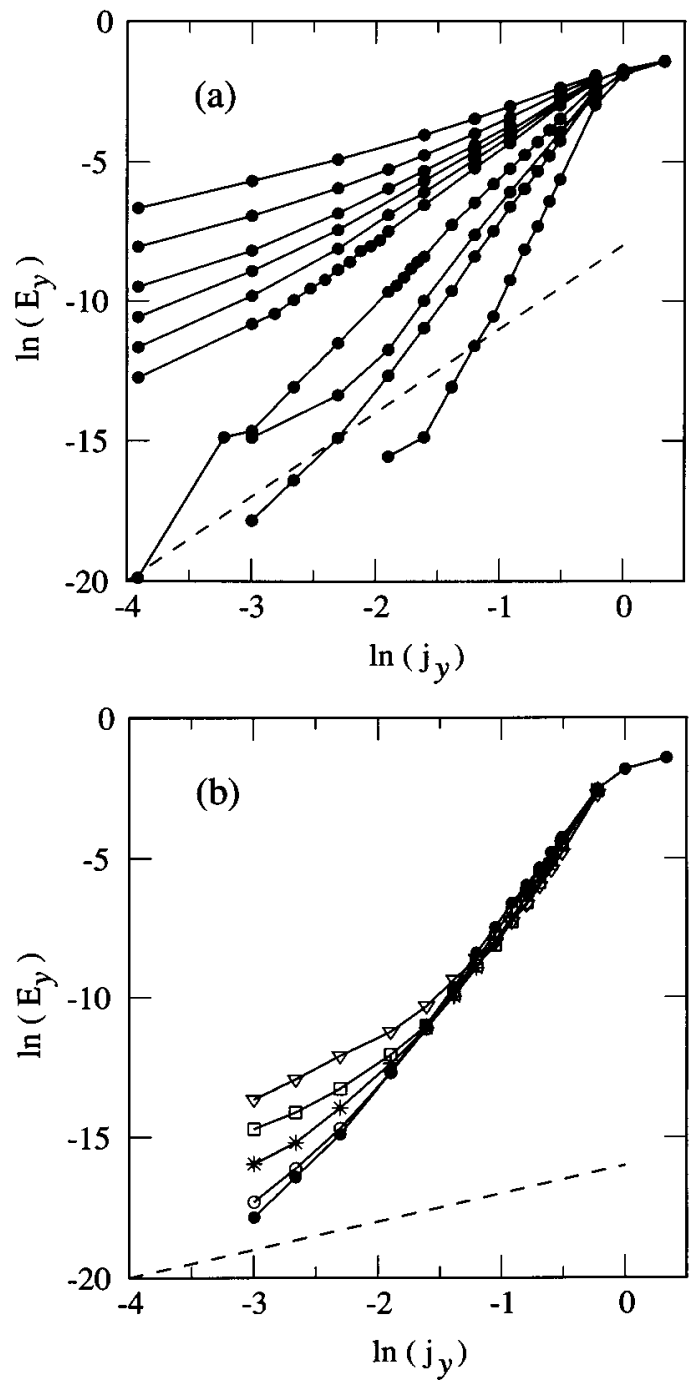

FIG. 1. Monte Carlo results for the nonlinear $I V$ characteristic of the two-dimensional lattice Coulomb gas. $E$ is the electrical field and $j$ is the supercurrent density. In (a) data shown are for parameters $L=32$ and $\mu=0.0$. Different curves are for different temperatures, starting from below $T=0.12,0.15,0.16,0.18,0.20,0.22$, $0.23,0.24,0.26$, and 0.30 . The dashed line has slope 3 which represents the Kosterlitz-Thouless transition. In (b) finite-size effects according to Eq. (26) are demonstrated. Data here is for $T=0.15$ and lattice sizes are $L=8$ (triangles), 12 (open squares), 16 (stars), 24 (open circles), and 32 (filled circles). The dashed line has slope 1 which represents the linear Ohmic regime.

pendent determination ${ }^{12}$ of $T_{c}$ for this system, based on the finite-size scaling relation Eq. (6).

In Fig. 1(b) we demonstrate the effects of the finite lattice size for low driving currents at temperatures below $T_{c}$. The data shown are for $T=0.15$ and lattice sizes are $L=8$ (triangles), 12 (open squares), 16 (stars), 24 (open circles), and 32 (filled circles).

The finite-size effects for the lower temperatures can be understood in the following way. [See the discussion above following Eq. (15).] The finite lattice size is important because pair excitation over the barrier given by the periodicity length $L$ will add to the dissipation due to unbinding of pairs over the barrier given by the pair size $r^{*}$. The induced electric field will accordingly be of the form

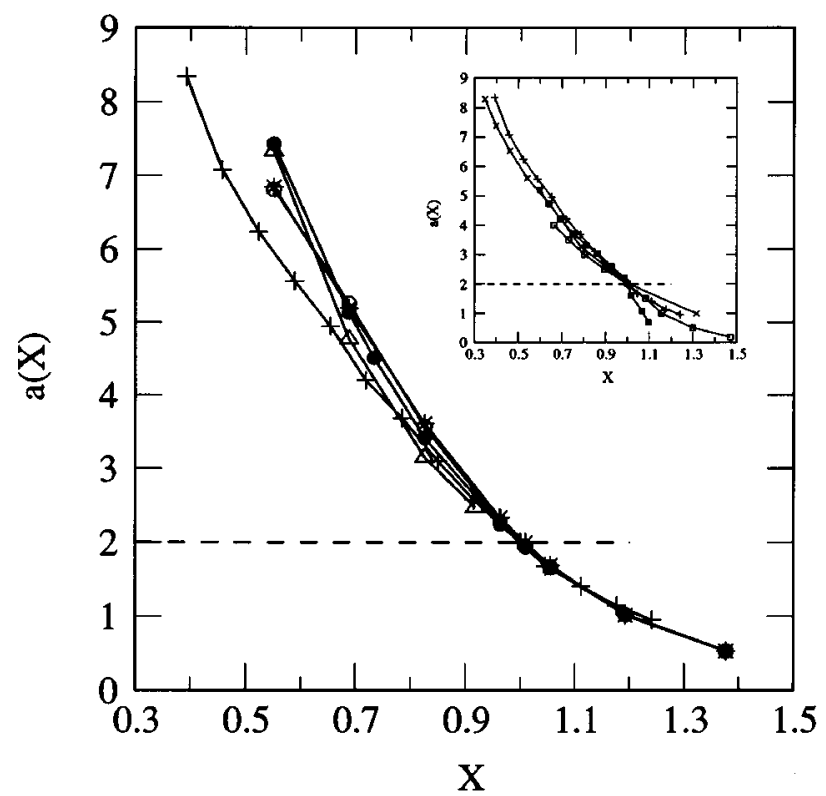

FIG. 2. Comparison of the exponent $a(X)$ between experiments, marked with plusses (Ref. 26) and Monte Carlo results for the lattice Coulomb gas Sizes are $L=16$ (stars), 24 (open circles), 32 (filled circles), and 48 (triangles). $X=T / T_{c}$ is the reduced Coulomb gas temperature. The dashed line corresponds to the universal jump condition for the exponent, $a(X)=2$. The inset shows experimental data for $\mathrm{Hg}$-Xe alloy films (plusses) (Refs. 4 and 26), $\mathrm{Bi}_{2} \mathrm{Sr}_{2} \mathrm{CaCu}_{2} \mathrm{O}_{x}$ single-crystal films (filled squares) (Ref. 7), and $\mathrm{Bi}_{1.6} \mathrm{~Pb}_{0.4} \mathrm{Sr}_{2} \mathrm{Ca}_{2} \mathrm{Cu}_{3} \mathrm{O}_{x}$ single-crystal films (open squares) (Ref. 8).

$$
E=R(L) j+\text { const }^{E_{1}} / 2 T+1,
$$

where the first term $R(L)$ follows from Eq. (16) and $R(L) \rightarrow 0$ as $L \rightarrow \infty$. The second term in Eq. (26) is given by Eq. (15) and will remain finite in the limit $L \rightarrow \infty$. This is clearly demonstrated in Fig. 1(b) where we see that the crossover in Eq. (26) between the linear and nonlinear regime appears at a higher driving current for the smaller $8 \times 8$ lattice as the current length $\left(E_{1} / I=\xi_{I} \sim L\right)$ associated with the current density $j$ exceeds the size of the lattice.

In Fig. 2 the exponent $a_{I V}(X)$ is shown as a function of the reduced temperature $X=T / T_{c}$. The dashed horizontal line represents the universal jump condition for $a_{I V}(X)$. The plusses represent experimental data from a superconducting $\mathrm{Hg}$-Xe film. ${ }^{4,26}$ The filled circles are the results for $a_{I V}(T)$ from Fig. 1. The other three data sets are for lattice sizes $L=16$ (stars), 24 (open circles), and 48 (triangles). As one can see there are no apparent finite-size effects in the data. In the vicinity of the critical temperature the experimental data are reproduced by the Monte Carlo simulations.

The reduced temperature variable $X$ used in Fig. 2 for the experiment is also from Ref. 26 and for the lattice Coulomb gas data we use $T_{c}=0.218,{ }^{12}$ determined from a finite-size scaling analysis using Eq. (6).

The inset in Fig. 2 shows a selection of experimental data analyzed along the lines described in Ref. 26. The data in the inset (plusses) are the same as in the main figure, the other data are for $\mathrm{Bi}_{2} \mathrm{Sr}_{2} \mathrm{CaCu}_{2} \mathrm{O}_{x}$ single crystal (filled squares), ${ }^{7}$ and for $\mathrm{Bi}_{1.6} \mathrm{~Pb}_{0.4} \mathrm{Sr}_{2} \mathrm{Ca}_{2} \mathrm{Cu}_{3} \mathrm{O}_{x}$ single crystal (open squares) ${ }^{8}$ 

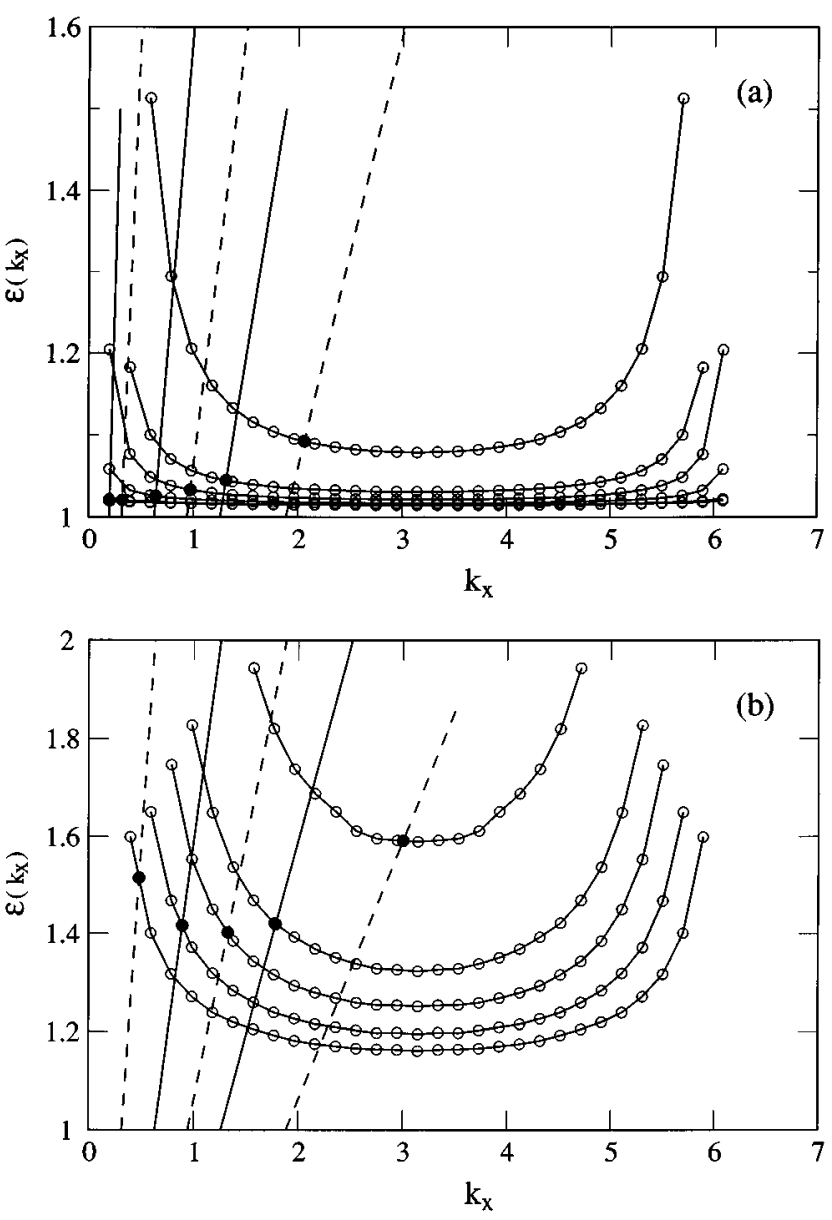

FIG. 3. Construction of the self-consistent solution. The data shown are for $T=0.18$ in (a) and $T=0.240$ in (b). The different curves represent $\epsilon(k)$ as a function of $k_{x}$ for different current densities $j=0.05$ (bottom curve), 0.10,0.15,0.20,0.30 (top curve). The straight lines represent the self-consistency condition for the different current densities, and the intersections of the straight lines with the $\epsilon(k)$ curve for the same current density $j$ represents the selfconsistent solution, here marked with large filled circles.

The Monte Carlo data presented here for $a(X)$ are all for $\mu=0.0$. We also did the same analysis for Monte Carlo data for $L=32$ and $\mu=-0.4,-0.2$ and 0.2 . The closest fit to the experimental results is produced by $\mu=0.0$. Results for different $\mu$ differ from the $\mu=0.0$ results, by that $\mu=0.2$ has a slightly larger derivative at $X=1$ and the smaller $\mu$ are correspondingly less steep.

\section{B. Self-consistent $I V$ characteristic}

In our second determination of the exponent $a(T)$ we will make use of the relations between $\epsilon^{*}$ and $a(T)$ in Eqs. (20) and (21). The analysis is based on the self-consistent solution of Eq. (19). For a given current density $j$, a set of $\epsilon(k)$ will be calculated for different temperatures. The self-consistent solution to Eq. (19) for $\epsilon^{*}$ is shown in Fig. 3, in (a) data for $T=0.18$ is shown and in (b) $T=0.24$. The solid and dashed straight lines represent $\epsilon^{*}=k^{*} / j 2 \pi$, given by Eq. (19), for different current densities $j$. The open circles represent $\epsilon\left(\mathbf{k}^{*}\right)$ as a function of $k$ for different current densities. The choice of the direction along which $\epsilon\left(\mathbf{k}^{*}\right)$ is probed is per-

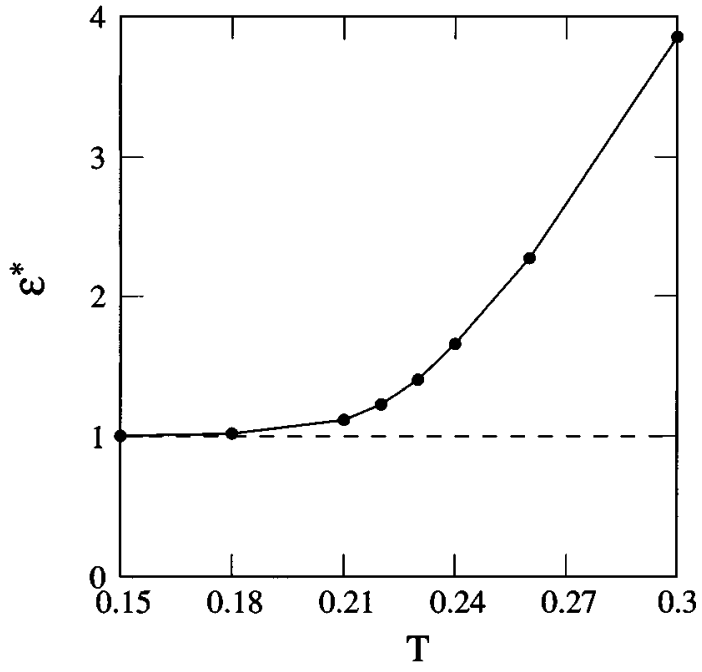

FIG. 4. The solution to the self-consistent equation (19). $\epsilon^{*}$ as a function of temperature for the fixed current density $j=0.03125$. Data shown are for size $L=32$ chemical potential $\mu=0.0$. The dashed line represents the bare $\epsilon=1$.

pendicular to the current density $j$, i.e., parallel to the vortex drift caused by the current density $j$. The intersection between a $\epsilon\left(\mathbf{k}^{*}\right)$ curve and the corresponding straight line is the solution to Eq. (19), these are marked with filled circles. In Fig. 3(a) for $T=0.18<T_{c}=0.218$ we see that the selfconsistent solution depends only weakly on the choice of probing current as long as the current is not too large. In Fig. 3(b) for $T=0.24>T_{c}$ we see, however, that there is no well defined limiting solution for $\epsilon^{*}$ as $j \rightarrow 0$. This is because the system is above the Kosterlitz-Thouless temperature and vortex pairs will always dissociate irrespective of $j$. In Fig. 4 the function $\epsilon^{*}$ is shown as a function of temperature. The solid circles represent $\epsilon^{*}$ from the self-consistent Eq. (19) for the fixed current density $j=0.03125$. The data shown here represent the construction shown in Fig. 3.

The results from the self-consistent solution for $\epsilon^{*}$ are analyzed in Fig. 5. Here the filled circles represent the exponent $a_{I V}(T)$ from Fig. 2. The upside down triangles represent $a_{\mathrm{AHNS}}(T)$ from Eq. (20) with the solution from Fig. 3 and the triangles are the corresponding solution to Eq. (21). One can clearly see that the expression in Eq. (21), derived by Minnhagen et al., ${ }^{21}$ reproduces the exponent $a_{I V}(T)$ for $T<T_{c}$. Note however, it is only a coincidence that Eq. (20), derived by Ambegoakar et al., ${ }^{17}$ works for temperatures above $T_{c}$ in this figure as the limiting $(j \rightarrow 0)$ solution for $1 / \epsilon^{*}$ is not well defined for these temperatures, as already discussed in connection with Fig. 3(b). As the simulation data $a_{I V}(T)$ (filled circles) also matched the experimental data in Fig. 1 we must conclude that below $T_{c}$ the interpretation according to Eq. (21) is clearly the more appropriate.

\section{Linear resistance}

We will now turn to our last determination of the exponent $a(T)$. The results presented above all relied on nonequilibrium Monte Carlo simulations, i.e., with a finite applied supercurrent density $j$. We will now present the equilibrium determination for $j=0$ based on finite-size scaling of Monte 


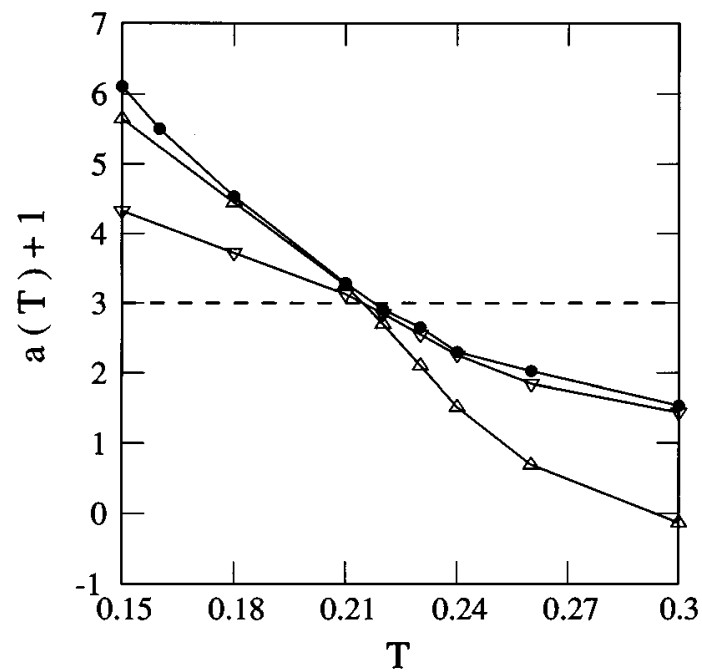

FIG. 5. The $I V$ exponent $a(T)$ as a function of temperature. Data shown are for size $L=32$ and chemical potential $\mu=0.0$. The filled circles are $a_{I V}(T)$ from the the nonlinear $I V$ characteristic shown in Fig. 1(b). The upside down triangles are the exponent $a_{\text {AHNS }}(T)$ from Eq. (20) using $\epsilon^{*}$ from Fig. (4). The triangles represent the exponent $a_{\mathrm{PM}}(T)$ from Eq. (21) also using $\epsilon^{*}$ from Fig. 4.

Carlo data for the linear resistance given by the Nyquist formula (5) together with Eq. (17). In Fig. 6 we demonstrate a data collapse of the linear resistance for several lattice sizes. From Eq. (17) we see that the linear resistance data can be collapsed onto a single curve, thus representing the thermodynamic limit, by an appropriate choice at each temperature $T$ of the exponent $a_{R}(T)$. We do this in the following way. For a given temperature we find the exponent $a_{R}$ which minimizes the error of the fit defined as $\Sigma_{L, L^{\prime}}\left[R(L) L^{a}-R\left(L^{\prime}\right) L^{\prime a}\right]^{2}$. The considered lattice sizes

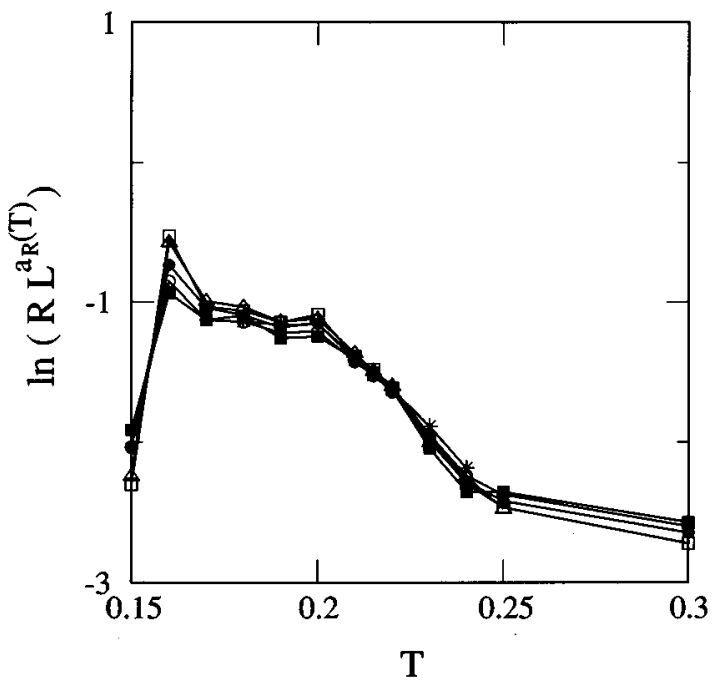

FIG. 6. Finite-size scaling of the linear resistance $R$. The resistance has been calculated with the Nyquist formula (5). Shown is a data collapse of $R L^{a_{R}(T)}$ as a function of $T$ for different lattice sizes, $L=6,8,12,16,24,32$. The exponent $a_{R}(T)$ is chosen in such a way as to provide the best data collapse. According to Eq. (17) the function $R L^{a_{R}(T)}$ should be independent of system size.

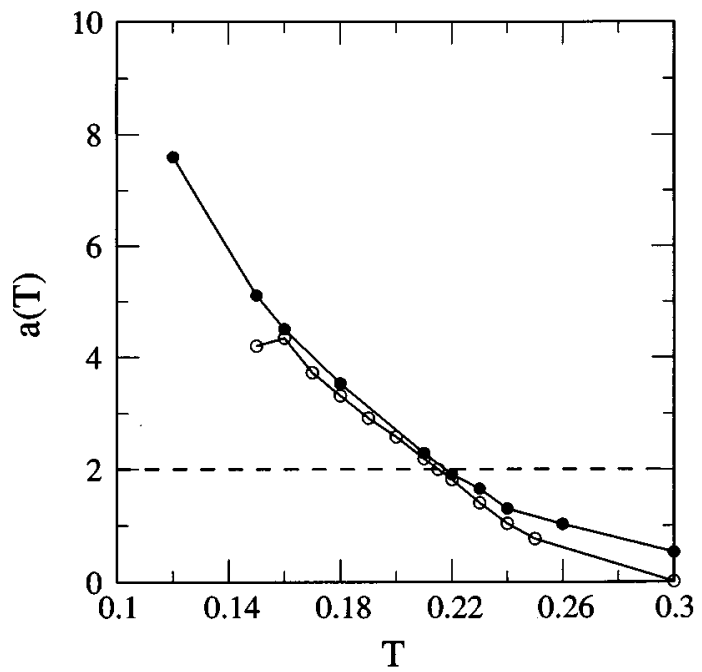

FIG. 7. Comparison of the exponent $a(T)$ as a function of temperature $T$, from two different determinations. The filled circles represent the exponent $a_{I V}(T)$ determined in Fig. 2 from the nonlinear $I V$ characteristics. The open circles are results for $a_{R}(T)$ from Fig. 6 from finite-size scaling of the linear resistance.

are $L, L^{\prime}=6,8,12,16,24,32$. The obtained scaling exponent $a_{R}$ as a function of temperature is shown in Fig. 7. As a comparison we also show data for $a_{I V}(T)$ from Fig. 2, obtained from direct evaluation of the $I V$ characteristic. The finite-size scaling analysis in Fig. 6 breaks down for low temperatures. This can be seen by the deviation of $a_{R}(T)$ from the data for $a_{I V}(T)$ at $T=0.15$. In Fig. 7 this deviation is also evident. A careful inspection of the scaling at temperatures $T=0.15$ and $T=0.18$ reveals that the order of the lattices sizes is reversed for $T=0.15$ compared with the higher temperatures. This may be related to the difficulties to converge the simulation at low temperature.

\section{DISCUSSION}

We have calculated the nonlinear $I V$ exponent $a_{I V}(T)$ of the two-dimensional lattice Coulomb gas. Our results are based on three different determinations. A direct calculation of the voltage response as a function of an applied current. Comparison with experiments ${ }^{4,7,8,26}$ on $\mathrm{Hg}$-Xe films and single-crystal high- $T_{c}$ superconductors show good agreement.

Our second method is based on a simple self-consistent calculation of the dielectric function $\epsilon^{*}$ at the unbinding separation, and the $I V$ exponent can then be calculated. Here we especially focus on the comparison of two relations between $a(T)$ and $\epsilon$. The first relation Eq. (20) (Ref. 17) is based on ordinary diffusion in two dimensions with a recombination rate proportional to $n_{F}^{2}$. The second expression for $a(T)$ given in Eq. (21) has been derived from a scaling analysis. $^{21}$

We find that the exponent determined by Eq. (21) for temperatures below $T_{c}$ is close to the more direct determined $a_{I V}(T)$ and will therefore also fit the experiments for these temperatures.

The third method is based on equilibrium Monte Carlo simulations. From the scaling relation Eq. (17) for the linear 
resistance we can derive $a_{R}(T)$. We find that the scaling exponent $a_{R}(T)$ to a high degree of accuracy fits the direct determined $a_{I V}(T)$ for a broad range of temperatures. This provides a link between the equilibrium and nonequilibrium response properties of the system: A finite mesoscopic linear resistance in a finite sample below $T_{c}$ is due to thermally activated vortex motion across some potential barrier, generated by interactions with all other vortices in the system. When a finite current is imposed across the system, new nonequilibrium configurations are accessed where vortices are driven away from their equilibrium positions by the finite Lorentz force, thus giving nonlinear response. Our data show that the barrier overcome by the Lorentz force, giving nonlinear response, is essentially the same barrier as in the equilibrium case, i.e., the potential barrier in the case of a finite current appears to be determined by equilibrium states in the system. This is consistent with the scaling ansatz, discussed above, of a certain current length scale $\left(r^{*}\right)$ associated with the finite current, such that for lengths shorter than the current length scale an equilibrium state is still attained, which gives a potential barrier essentially equal to that in the equilibrium case.

An interesting possibility arises here to measure finitesize effects on the linear resistance in lithographic Josephson-junction arrays. The idea would here to take advantage of finite-size roundings, rather than as usual want them to be as small as possible, and trying to fit experimental data on very small arrays to our finite-size scaling formulas. This would provide an unusual experimental test of finitesize scaling. It is also important to analyze the data in terms of the reduced temperature scale, as $T_{c}$ is sample dependent. According to Eq. (17) it should be possible to scale the linear resistance of samples of different sizes onto a single scaling function using the exponent $a(X)$ from the nonlinear $I V$ characteristics.

Our conclusions are (1) simulation of nonequilibrium vortex dynamics allow calculation of a lattice size independent $I V$ exponent $a(T)$ as a function of temperature $T$. (2) This curve for $a(T)$ agrees nicely with experiments in an interval around $T_{c}$, and this appears to be reported here for the first time. (3) This curve can be obtained from a simple phenomenological theory for the nonlinear $I V$ characteristic. (4) This curve can also be obtained from a simulation of the equilibrium vortex dynamics. This provides a useful link between driven diffusion and equilibrium dynamics of twodimensional vortex systems.

\section{ACKNOWLEDGMENTS}

We acknowledge stimulating discussions with P. Minnhagen and K. Holmlund. H.W. was supported by grants from Carl Trygger, M.W. was supported by grants from the Swedish Natural Science Research Council (NFR) and H.J.J. was supported by the British EPSRC.
${ }^{1}$ J. M. Kosterlitz and D. J. Thouless, J. Phys. C 5, L124 (1972); 6, 1181 (1973).

${ }^{2}$ V. L. Berezinskii, Zh. Éksp. Teor. Fiz. 61, 1144 (1971) [Sov. Phys. JETP 34, 610 (1972)].

${ }^{3}$ P. Minnhagen, Rev. Mod. Phys. 59, 1001 (1987).

${ }^{4}$ A.M. Kadin, K. Epstein, and A.M. Goldman, Phys. Rev. B 27, 6691 (1983).

${ }^{5}$ S. Teitel and C. Jayaprakash, Phys. Rev. B 27, 598 (1983).

${ }^{6}$ J. Villain, J. Phys. (Paris) 36, 581 (1975).

${ }^{7}$ I. G. Gorlova and Yu. I. Latyshev, Pis'ma Zh. Éksp. Teor. Fiz. 51, 197 (1990) [JETP Lett. 51, 224 (1990)].

${ }^{8}$ A. K. Pradhan, S. J. Hazell, J. W. Hodby, C. Chen, Y. Hu, and B. M. Wanklyn, Phys. Rev. B 47, 11374 (1993).

${ }^{9}$ D. R. Nelson and J. M. Kosterlitz, Phys. Rev. Lett. 39, 1201 (1977).

${ }^{10}$ M. Wallin and S. M. Girvin, Phys. Rev. B 47, 14642 (1993).

${ }^{11}$ R. A. Hyman, M. Wallin, M. P. A. Fisher, S. M. Girvin, and A. P. Young, Phys. Rev. B 51, 15304 (1995).

${ }^{12}$ M. Wallin and H. Weber, Phys. Rev. B 51, 6163 (1995).

${ }^{13}$ J.-R. Lee and S. Teitel, Phys. Rev. B 50, 3149 (1994).

${ }^{14}$ J. V. José, L. P. Kadanoff, S. Kirkpatrick, and D. R. Nelson, Phys. Rev. B 16, 1217 (1977).
${ }^{15}$ J.-R. Lee and S. Teitel, Phys. Rev. Lett. 64, 1483 (1990); Phys. Rev. B 46, 3247 (1992).

${ }^{16}$ F. Reif, Fundamentals of Statistical and Thermal Physics (McGraw-Hill, New York, 1965).

${ }^{17}$ V. Ambegoakar, B. I. Halperin, D. R. Nelson, and E. D. Siggia, Phys. Rev. Lett. 40, 783 (1978); Phys. Rev. B 21, 1806 (1980).

${ }^{18}$ M. Tinkham, Introduction to Superconductivity (McGraw-Hill, New York, 1975).

${ }^{19}$ H. Weber and P. Minnhagen, Phys. Rev. B 37, 5986 (1988).

${ }^{20}$ P. Olsson, Phys. Rev. B 46, 14598 (1992).

${ }^{21}$ P. Minnhagen, O. Westman, A. Jonsson, and P. Olsson, Phys. Rev. Lett. 74, 3672 (1995).

${ }^{22}$ N. Metropolis, A. W. Rosenbluth, M. N. Rosenbluth, A. H. Teller, and E. Teller, J. Chem. Phys. 21, 1087 (1953).

${ }^{23}$ A. P. Young, in Proceedings of the Ray Orbach Inauguration Symposium (World Scientific, Singapore, 1994).

${ }^{24}$ P. Meakin, H. Metiu, R. G. Petschek, and D. J. Scalapino, J. Chem. Phys. 79, 1948 (1983).

${ }^{25}$ J.-R. Lee and S. Teitel, Phys. Rev. B 46, 3247 (1993).

${ }^{26}$ P. Minnhagen, Phys. Rev. B 28, 2463 (1983). 